\title{
Cap-assisted large cold snare removal of a giant phytobezoar
}

A 56-year-old woman presented to our department with a 1-day history of hematemesis and a 2-year history of eating persimmons. After the patient had received an injection of proton pump inhibitor, we found a giant phytobezoar (about $6 \times 4 \mathrm{~cm}$ ) in her stomach on gastroscopy ( $\triangleright$ Fig.1a). The phytobezoar was turned into an upright position with the endoscope in the inverted position ( Video 1$)$. A large snare (4cm in diameter) was used to trap the phytobezoar ( Fig. 1 b); however, it was hard to crush the phytobezoar when tightening the snare. Therefore, the tightened snare was pulled into the cap to cold-cut the phytobezoar using cap assistance. After repeated snare-trapping and cap-assisted cold-cutting, the phytobezoar was finally cut into several pieces. The larger pieces were pulled out using the snare ( Fig.2); the leftover small pieces were washed out of the body using oral polyethylene glycol. A subsequent gastroscopy, 2 days after the cap-assisted coldcutting snare removal procedure, revealed a clear stomach (\$Fig. $\mathbf{3}$ ).

Gastric bezoars can be removed by drinking coco-cola, or the use of forceps, snare, or DualKnife [1,2]; however, a giant gastric bezoar is difficult to remove. A previous report described cap-assisted cold snare removal of a small cyanoacrylate glue bezoar [3]. Herein, we show that a cap-assisted cold snare technique can also be used to easily remove giant phytobezoars with large diameters.

Endoscopy_UCTN_Code_TTT_1AO_2AL

Funding

the Bureau of Science \& Technology Nanchong City No. 18 SXHZ0466

Competing interests

The authors declare that they have no conflict of interest.

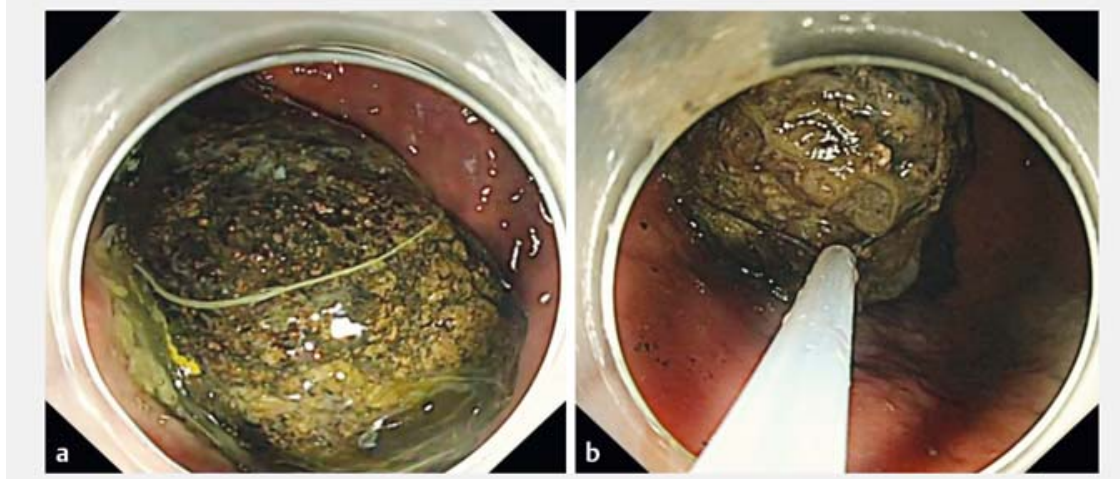

Fig. 1 Endoscopic views showing: a a giant phytobezoar (about $6 \times 4 \mathrm{~cm}$ ) in the stomach; b a large cold snare being used to trap the phytobezoar.

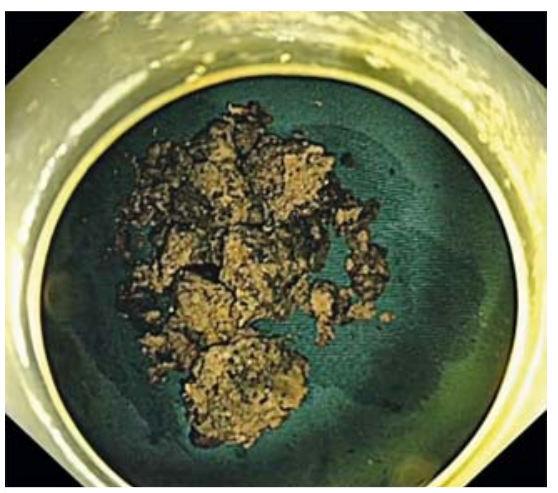

Fig. 2 Photograph of the some of the larger pieces of the phytobezoar that were removed with the snare.

The authors

Zhang Tao Wenfeng Pu, Liang Sun, Xiaoqing Zhou, Yan Zhang, Zhonghan Du, Ji Wu

Department of Gastroenterology, Nanchong Central Hospital, Nanchong City, Sichuan, China

\section{Corresponding author}

\section{Ji Wu, MD}

Department of Gastroenterology, Nanchong Central Hospital, Nanchong City, Sichuan, 637000, China

wuji2168@sina.com

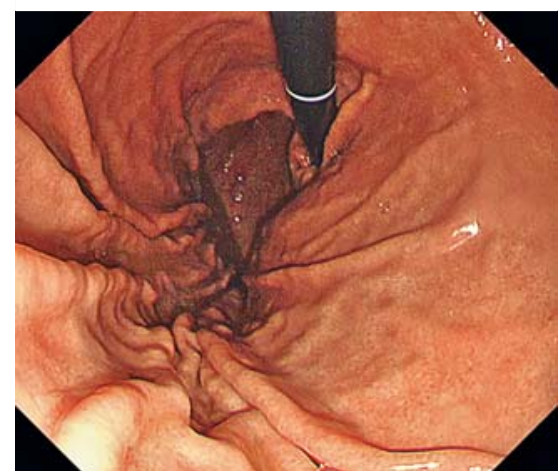

- Fig. 3 Image from a repeat endoscopy 2 days after the cold snare excision showing a clear stomach.

References

[1] Iwanuro M, Okada H, Matsueda K et al. Review of the diagnosis and management of gastrointestinal bezoars. World J Gastrointest Endosc 2015; 7: 336-345

[2] Tao Z, Yu Y, Zhou X. New application of dual knife: Easier removal of a giant bezoar. Dig Endosc 2019; 31: e62-e63

[3] Sun M, Pan S, Liang Y et al. Removal of a duodenal cyanoacrylate glue bezoar with a snare and cap-assisted endoscopy. Endoscopy 2020. doi:10.1055/a-1244-9779 


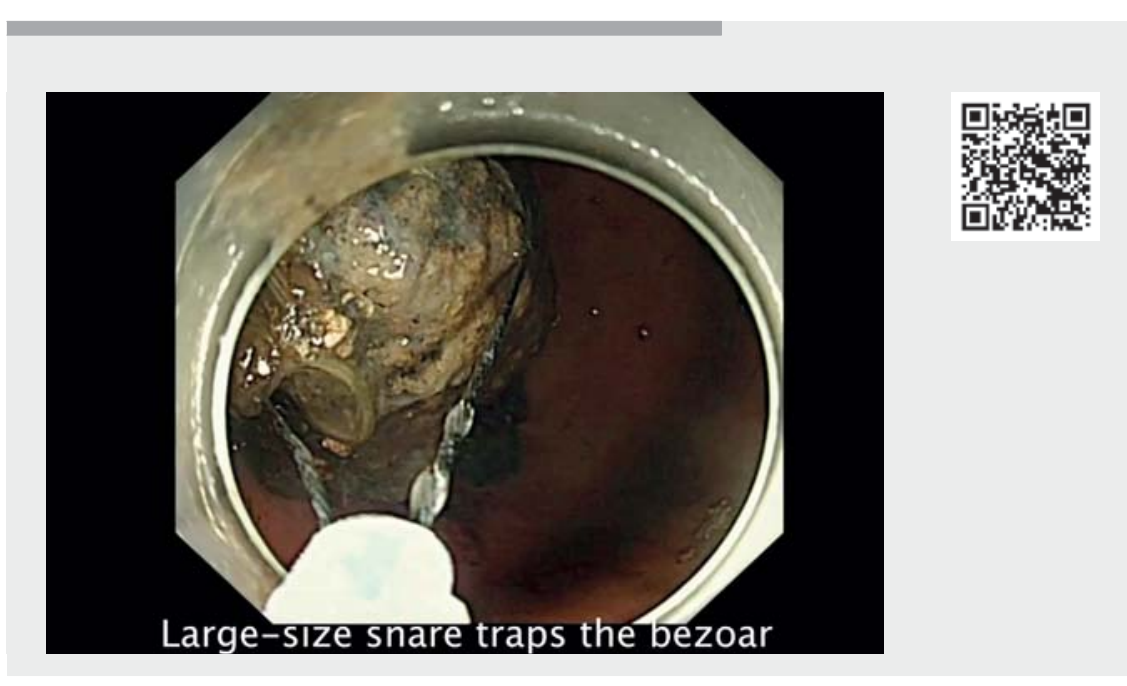

$\checkmark$ Video 1 A giant phytobezoar is removed using a repeated cap-assisted large cold snare technique to fragment the bezoar, with the larger pieces being removed with the snare, leaving the stomach clear after flushing with oral polyethylene glycol.
Bibliography

Endoscopy 2022; 54: E222-E223

DOI $10.1055 / \mathrm{a}-1492-1874$

ISSN 0013-726X

published online 31.5.2021

(C) 2021. Thieme. All rights reserved.

Georg Thieme Verlag KG, Rüdigerstraße 14, 70469 Stuttgart, Germany

\section{ENDOSCOPY E-VIDEOS}

https://eref.thieme.de/e-videos

回回 Endoscopy E-Videos is an open access online section, 回证: reporting on interesting cases and new techniques in gastroenterological endoscopy. All papers include a high quality video and all contributions are freely accessible online. Processing charges apply (currently EUR 375), discounts and wavers acc. to HINARI are available.

This section has its own submission website at

https://mc.manuscriptcentral.com/e-videos 